\title{
Pulmonary Oligometastases Treated by Stereotactic Body Radiation Therapy: A Nationwide Survey of 1,378 Patients
}

\author{
YUZURU NIIBE ${ }^{1,2}$, TAKAYA YAMAMOTO ${ }^{3}$, HIROSHI ONISHI ${ }^{4}$, HIDEOMI YAMASHITA ${ }^{5}$, KUNIAKI KATSUI $^{6}$, \\ YASUO MATSUMOTO ${ }^{7}$, RYOONG-JIN OH ${ }^{8}$, MASAHIKO AOKI ${ }^{9}$, TAKASHI SHINTANI ${ }^{10}$, KAZUNARI YAMADA ${ }^{11}$, \\ MITSURU KOBAYASHI ${ }^{12}$, MASATOKI OZAKI ${ }^{13}$, YOSHIHIKO MANABE ${ }^{14}$, KATSUYA YAHARA $^{15}$, \\ ATSUSHI NISHIKAWA ${ }^{16}$, HISAO KAKUHARA ${ }^{17}$, KENTARO YAMAMOTO $^{18}$, TETSUYA INOUE $^{19}$, YU TAKADA ${ }^{20}$, \\ KENJI NAGATA $^{21}$, OSAMU SUZUKI ${ }^{22}$, ATSURO TERAHARA ${ }^{1}$ and KEIICHI JINGU ${ }^{3}$ \\ ${ }^{1}$ Department of Radiology, Toho University Omori Medical Center, Tokyo, Japan; \\ ${ }^{2}$ Department of Primary Care and Medical Education, Okayama University, Okayama, Japan; \\ ${ }^{3}$ Department of Radiation Oncology, Tohoku University Graduate School of Medicine, Sendai, Japan; \\ ${ }^{4}$ Department of Radiology, University of Yamanashi, Yamanashi, Japan; \\ ${ }^{5}$ Department of Radiology, the University of Tokyo, Tokyo, Japan; \\ ${ }^{6}$ Department of Proton Beam Therapy, Okayama University, Okayama, Japan; \\ ${ }^{7}$ Department of Radiation Oncology, Niigata Cancer Center Hospital, Niigata, Japan; \\ ${ }^{8}$ Department of Radiation Oncology, Miyakojima IGRT Clinic, Osaka, Japan; \\ ${ }^{9}$ Department of Radiology, Hirosaki University, Hirosaki, Japan; \\ ${ }^{10}$ Department of Radiation Oncology and Image-applied Therapy, Kyoto University, Kyoto, Japan; \\ ${ }^{11}$ Department of Radiation Oncology, Keiyukai Sapporo Hospital, Sapporo, Japan; \\ ${ }^{12}$ Department of Radiation Oncology, Seirei Mikatahara General Hospital, Shizuoka, Japan; \\ ${ }^{13}$ Deparment of Radiation Oncology, Fukuyama City Hospital, Hiroshima, Japan; \\ ${ }^{14}$ Department. of Radiation Oncology, Shizuoka City Shimizu Hospital, Shizuoka, Japan; \\ ${ }^{15}$ Department of Radiology, Nagoya City University, Nagoya, Japan; \\ ${ }^{16}$ Department of Radiology, University of Occupational and Environmental Health, Fukuoka, Japan; \\ ${ }^{17}$ Department of Radiation Oncology, Shikoku Cancer Center, Ehime, Japan; \\ ${ }^{18}$ Department of Radiology, Iwate Medical University, Iwate, Japan; \\ ${ }^{19}$ Department of Radiology, Self-Defense Forces Central Hospital, Tokyo, Japan; \\ ${ }^{20}$ Department of Nagasaki Prefecture Shimabara Hospital, Nagasaki, Japan; \\ ${ }^{21}$ Department Radiology, Hokkaido University Hospital, Sapporo, Japan; \\ ${ }^{22}$ Department of Radiology, Juntendo University Hospital, Tokyo, Japan
}

\begin{abstract}
Aim: This study was performed to confirm the superior overall survival (OS) after pulmonary oligo-recurrence compared to pulmonary sync-oligometastases in a large nationwide study. Patients and Methods: Patients that met the following criteria were included: 1 to 5 lung-only metastases at the beginning of stereotactic body radiation therapy (SBRT) was
\end{abstract}

Correspondence to: Yuzuru Niibe, MD, Ph.D., Department of Primary Care and Medical Education, Okayama University, MUSCAT CUBE 2F, Shikata Campus, 2-5-1, Shikata-cho, Kita-ku, Okayama, 700-8558, Japan. Tel: +81 862356963, Fax: +81 862356834, e-mail: joe-n@hkg.odn.ne.jp

Key Words: Pulmonary oligometastases, pulmonary oligo-recurrence, pulmonary sync-oligometastases, SBRT, nationwide study. performed between January 2004 and June 2015, and the biological effective dose (BED) of SBRT was 75 Gy or more. The parameters included in the analyses were age, gender, ECOG PS, primary lesion, pathology, oligoetastatic state, SBRT date, chemotherapy before SBRT, chemotherapy concurrent $S B R T$, chemotherapy after SBRT, maximum tumor diameter, number of metastases, field coplanarity, dose prescription, $B E D_{10}$, OTT of SBRT. Results: In total, 1,378 patients with 1,547 tumors were enrolled. Oligo-recurrence occurred in 1,016 patients, sync-oligometastases in 118, and unclassified oligometastases in 121. The three-year OS was $64.0 \%$ for oligorecurrence and $47.5 \%$ for sync-oligometastasis $(p<0.001)$. In the multivariate analysis, the hazard ratio (HR) for syncoligometastases versus oligo-recurrence was 1.601 ( $p=0.014)$. Adverse events of Grade 5 were occurred in 3 patients. Conclusion: This is the first nationwide to indicate that the OS 
of patients with pulmonary oligo-recurrence is better than that of patients with sync-oligometastases.

Distant metastasis or distant recurrence at any site or in any organ was considered to indicate the terminal stage of cancer before the 2010s. However, this above status is not always indicative of the terminal stage in cancer patients. In particular, between 1995 and the early 2010s, the status of five or less metastases or recurrences was introduced and classified as oligometastases, oligo-recurrence, or syncoligometastases (1-3). The investigation of oligometastases has since progressed, and currently, the term is used to denote 1 to 5 metastases or recurrences in patients with cancer, regardless of whether the primary lesions are controlled. These metastases or recurrences are treated by local therapy including surgery, radiotherapy, or radiofrequency ablation and other local therapies (2-4).

Nowadays, oligometastases is classified into two statuses: The first is oligo-recurrence, which refers to cancer patients with 1 to 5 recurrences that are treated by local therapy, with controlled primary lesions (2-4). The other status is called sync-oligometastases and refers to cancer patients with 1 to 5 recurrences and active primary lesions, all treated by local therapy (3, 4). In 2016, Niibe et al. first reported that when limited to lung and/or brain non-small cell lung cancer (NSCLC), oligo-recurrence achieved a better overall survival (OS) than sync-oligometastases, although only 61 patients were included in that study because of rare occurrence (4). Likewise, 96 lung oligometastases patients were investigated in 2016 (5), and this larger population study reported the same results.

However, we do not consider 100 patients to be a sufficient sample size; therefore, larger nationwide population database studies are required. Thus, the primary purpose of this study was to statistically reveal the OS superiority of lung oligorecurrence compared to lung sync-oligometastases using univariate and multivariate analyses and to evaluate this comparison quantitatively using hazards ratios (HRs). The primary endpoint of this large database study was to determine the superiority or inferiority HR of sync-oligometastases vs. oligo-recurrence in a multivariate analysis.

\section{Patients and Methods}

Patient eligibility. The patient inclusion criteria were as follows: Patients with 1 to 5 lung metastases at the start of stereotactic body radiation therapy (SBRT) targeting the tumors (regardless of whether the primary lesions were controlled) were included. However, because patients under the Japanese Social Insurance System were included in this study, the primary lesion was controlled and other detectable lesions were limited in the lungs before beginning SBRT. The SBRT was performed between January 2004 and June 2015, and the biological effective dose (BED) of SBRT was $\geq 75$ Gy with a dose per fraction of $\geq 4$ Gy. BED 10 was calculated using the following formula: $B E D=n d[1+d /(\alpha / \beta)]$, where $\mathrm{n}$ is the number of fractions, $\mathrm{d}$ is the dose per fraction, and the $\alpha / \beta$ ratio is applied for $10 \mathrm{~Gy}$ for the tumors. Pulmonary metastases were defined as the appearance of a solid tumor in the lung simultaneously or after treatment of the primary tumor; local recurrence of a thoracic primary tumor was excluded.

Data acquisition and opt-out consents. For the purpose of the retrospective, observational, multicenter study, recruitment questionnaire was sent to the institutions registered in the Japanese Society for Radiation Oncology (JASTRO) database. Among the 426 institutions, 83 joined, but 15 had no eligible patients or later refused to participate. Finally, this study was conducted in 68 institutions in Japan. This study was approved by the Ethics Committee of Toho University Omori Medical Center, reference number, (reference number: 27-148), and all participating institutions guaranteed the chance to opt-out of participation in this study by providing information of this study via the internet or posters. Opt-out consents were obtained from all included patients.

Definitions. The disease-free interval (DFI) was defined as the interval between the date that the primary lesion was controlled and the date that the first metastasis was confirmed. The DFI start date was the date that surgery or radiofrequency ablation, etc., was performed, or the last date of radiotherapy or particle therapy. Adjuvant therapy (chemotherapy or hormonal therapy) was not considered. The DFI of oligo-recurrences, sync-oligometastases, and unclassified oligometastases was defined as a DFI $\geq 6,0$, and $<6$ months, respectively. Local failure was defined as enlargement of the irradiated tumor, and local control (LC) was defined as freedom from local failure. Relapse-free survival (RFS) was defined as freedom from any metastases, any recurrences, or death.

Statistical analysis. The time to an event was calculated from the first day of SBRT to the day an event was confirmed. The KaplanMeier method was used to calculate the cumulative LC rate, RFS rates, and OS rates, and Greenwood's formula was used to calculate 95\% confidence intervals (95\% CIs). A log-rank test was used to compare Kaplan-Meier curves. The Cox proportional hazards model was used to perform the multivariate analyses. A stepwise backward elimination/forward addition approach using the Akaike information criterion was applied to build the best multivariate analysis model. A $p$-value less than 0.05 was defined as significant. EZR, version 1.37 (Saitama Medical Center, Jichi Medical University, Saitama, Japan), a modified version of $\mathrm{R}$ commander ( $\mathrm{R}$ Foundation for Statistical Computing, Vienna, Austria), was used for the analyses (6). The National Cancer Institute Common Terminology Criteria for Adverse Events version 4.0 was used to grade adverse events.

\section{Results}

Acquisition results and treatment results. A total of 1,378 patients with 1,547 tumors were enrolled in this study. This large database study included all registered patients with or without missing values to avoid selection bias. The characteristics of the patients and tumors are listed in Table I. The oligometastatic states were distributed as follows: oligorecurrence in 1,016 patients, sync-oligometastases in 118 patients, and unclassified oligometastases in 121 patients. The median follow-up period for all patients was 24.2 months 
(range=0.1-143.7) and that for living patients was 26.9 months (range=0.1-143.7). During follow-up, 375 patients died from the primary disease, 109 died from other causes, and 52 died from unknown causes. There were 222 local failures, and the median interval to local failure was 12.4 months (range $=3.0$ 98.7). The 1-, 3-, and 5-year OS rates were 90.1\% (95\% $\mathrm{CI}=88.3 \%-91.6 \%), 60.3 \% \quad(95 \% \quad \mathrm{CI}=57.1 \%-63.3 \%)$, and $45.5 \%$ (95\% CI=41.8\%-49.1\%), respectively (Figure 1). The 1-, 3-, and 5-year RFS rates were 55.2\% (95\% CI=52.4\%$57.9 \%), 28.3 \%$ (95\% CI=25.7\%-31.0\%), and $19.5 \%(95 \%$ $\mathrm{CI}=16.9 \%-22.3 \%)$, respectively. The median OS and the median RFS were 51.4 months $(95 \% \mathrm{CI}=45.0-55.7)$ and 14.2 months (95\% CI=12.7-15.7), respectively. The 1-, 3-, and 5year $\mathrm{LC}$ rates were $92.1 \%$ (95\% $\mathrm{CI}=90.4 \%-93.4 \%), 81.3 \%$ (95\% $\mathrm{CI}=78.8 \%-83.6 \%$ ), and $78.6 \%$ (95\% $\mathrm{CI}=75.6 \%-81.2 \%)$, respectively. Adverse events were reported in 1,040 patients, and grade 2, 3, 4, and 5 pneumonitis occurred in $96(9.2 \%)$, $14(1.3 \%), 2(0.1 \%)$, and $7(0.6 \%)$ patients, respectively. Grade 5 hemoptysis occurred in 3 patients $(0.2 \%)$.

Univariate and multivariate analyses. In the univariate analyses (log-rank tests), significant differences in OS were found according to gender $(p<0.001)$, Eastern Cooperative Oncology Group performance status (ECOG PS, $p<0.001$ ), primary lesion $(p<0.001)$, pathology $(p<0.001)$, oligometastatic state $(p<0.001)$, chemotherapy after SBRT $(p=0.033)$, and maximum tumor diameter $(p<0.001)$ (Table II). The three-year OS was $64.0 \%(95 \% \mathrm{CI}=60.3 \%-67.4 \%)$ for oligo-recurrence, $47.5 \%(95 \% \mathrm{CI}=36.2 \%-58.0 \%)$ for sync-oligometastases, and $53.3 \%$ (95\% CI=42.6\%-62.8\%) for unclassified oligometastases.

The results of the multivariate analyses (Cox regression) are shown in Figure 2. The following factors were revealed to significantly negatively affect OS: ECOG PS 1 (PS 1 versus $\mathrm{PS} 0, \mathrm{HR}=1.415,95 \% \mathrm{CI}=1.116-1.795, p=0.004)$, ECOG PS 2-3 (PS 2-3 versus PS 0, HR=2.186, 95\% $\mathrm{CI}=1.469-3.253, p<0.001)$, primary lesion pathology of squamous cell carcinoma (squamous cell carcinoma versus adenocarcinoma, $\mathrm{HR}=1.440,95 \% \quad \mathrm{CI}=1.024-2.026$, $p=0.036$ ), primary lesion of esophagus (esophagus versus colorectum, $\mathrm{HR}=1.676,95 \% \mathrm{CI}=1.003-2.801, p=0.048$ ), sync-oligometastases (sync-oligometastases versus oligorecurrence, $\mathrm{HR}=1.601,95 \% \mathrm{CI}=1.097-2.336, p=0.014$ ), unclassified oligometastases (unclassified oligometastases versus oligo-recurrence, $\mathrm{HR}=1.457,95 \% \mathrm{CI}=1.039-2.043$, $p=0.029)$, and maximum tumor diameter $(\geq 1.5 \mathrm{~cm}$ versus $<1.5 \mathrm{~cm}, \mathrm{HR}=1.447,95 \% \mathrm{CI}=1.151-1.819, p=0.001)$.

\section{Discussion}

This retrospective nationwide study was selected by the JASTRO. As the primary endpoint, this study hypothesized that the HR for OS in patients with oligo-recurrence was superior to
Table I. Patient and treatment characteristics.

\begin{tabular}{|c|c|}
\hline Factors & $\begin{array}{c}\mathrm{n}(\%) \\
\mathrm{N}=1,378\end{array}$ \\
\hline \multicolumn{2}{|l|}{ Gender } \\
\hline Male & $894(64.9)$ \\
\hline Female & $484(35.1)$ \\
\hline \multicolumn{2}{|l|}{ Age (years) } \\
\hline Median, range & $72,16-93$ \\
\hline \multicolumn{2}{|l|}{ ECOG performance status } \\
\hline 0 & $744(56.3)$ \\
\hline 1 & 475 (35.9) \\
\hline $2-3$ & $103(7.8)$ \\
\hline \multicolumn{2}{|l|}{ Clinical stage; pathological stage } \\
\hline 1 & 187 (29.4); $222(35.9)$ \\
\hline 2 & $130(20.4) ; 133(21.5)$ \\
\hline 3 & $162(25.5) ; 173(28.0)$ \\
\hline 4 & 157 (24.7); 90 (14.6) \\
\hline \multicolumn{2}{|l|}{ Primary lesion } \\
\hline Lung & $422(30.6)$ \\
\hline Colorectum & $345(25.0)$ \\
\hline Head and neck & $114(8.3)$ \\
\hline Esophagus & $114(8.3)$ \\
\hline Others & $383(27.8)$ \\
\hline \multicolumn{2}{|l|}{ Pathology } \\
\hline Adenocarcinoma & $760(55.8)$ \\
\hline Squamous cell carcinoma & $392(29.3)$ \\
\hline Others & $185(13.8)$ \\
\hline \multicolumn{2}{|l|}{ Oligometastatic state } \\
\hline Oligo-recurrences & $1016(81.0)$ \\
\hline Sync-oligometastases & $118(9.4)$ \\
\hline Unclassified oligometastases & $121(9.6)$ \\
\hline \multicolumn{2}{|l|}{ DFI (months) median, range } \\
\hline Oligo-recurrences & $23.2,6.0-424$ \\
\hline Sync-oligometastases & 0 \\
\hline Unclassified oligometastases & $3.8,0.3-5.9$ \\
\hline \multicolumn{2}{|l|}{ Chemotherapy before SBRT } \\
\hline Yes & $503(36.8)$ \\
\hline No & $865(63.2)$ \\
\hline \multicolumn{2}{|l|}{ Chemotherapy concurrent SBRT } \\
\hline Yes & $29(2.1)$ \\
\hline No & $1349(97.9)$ \\
\hline \multicolumn{2}{|l|}{ Chemotherapy after SBRT } \\
\hline Yes & $190(17.7)$ \\
\hline No & $882(82.3)$ \\
\hline
\end{tabular}

Eastern Cooperative Oncology Group; DFI, disease-free interval; SBRT, stereotactic body radiotherapy.

that of patients with sync-oligometastases. The HR was 1.601, suggesting that our hypothesis was supported in this nationwide survey. Furthermore, the 1-, 3-, and 5-year OS rates of 90.1\%, $60.3 \%$, and $45.5 \%$, respectively, and the limited adverse events indicated that this technique of using BED in SBRT was effective and feasible for pulmonary oligometastases.

In a previous study investigating a similar hypothesis, Niibe et al. reported that the 2-year OS of lung oligo-recurrence was significantly higher than that of sync-oligometastases $(68.8 \%$ 


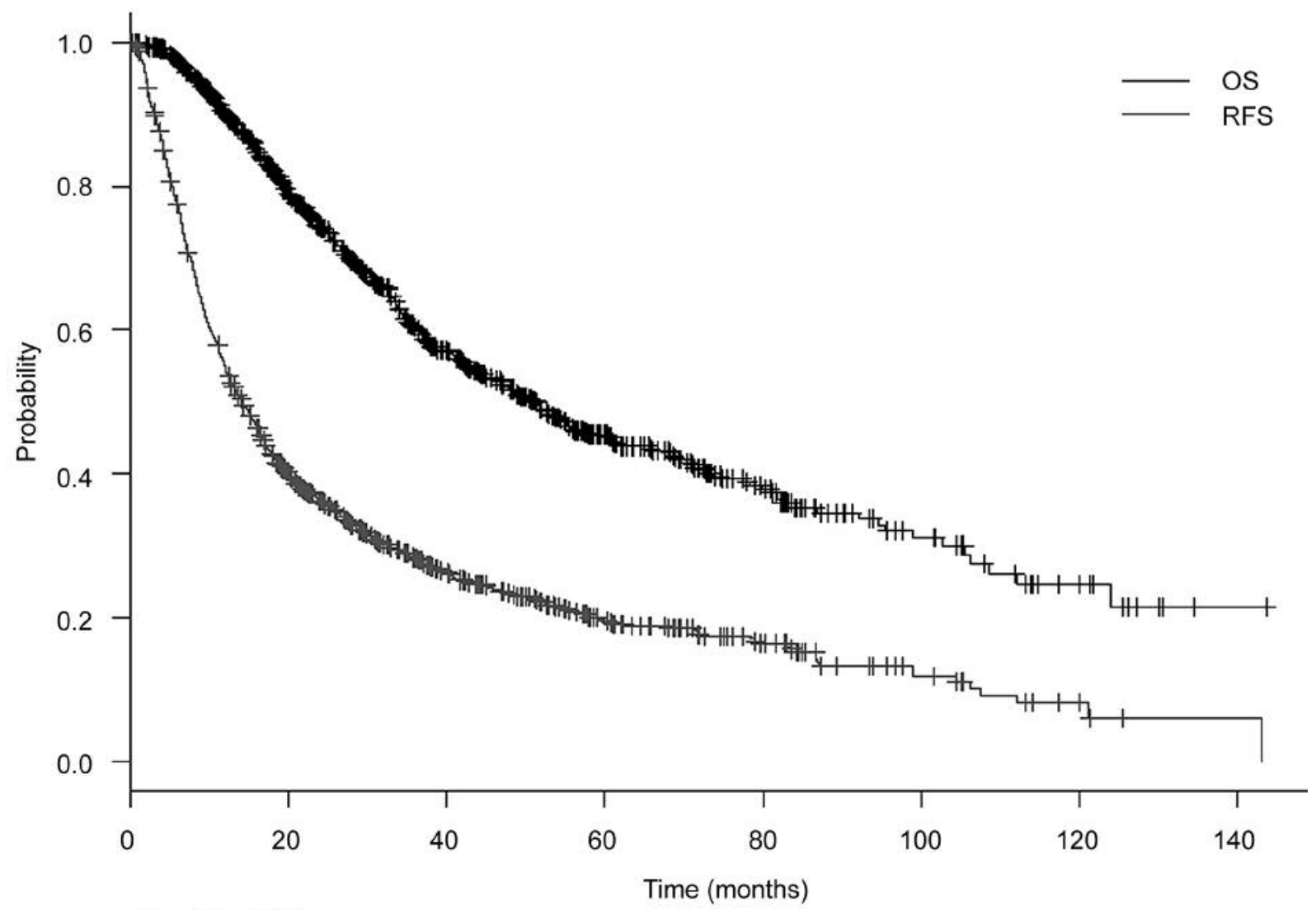

Number at risk

$\begin{array}{ccccccccr}\text { OS } & 1374 & 805 & 376 & 174 & 81 & 33 & 11 & 1 \\ \text { RFS } & 1331 & 436 & 193 & 93 & 46 & 18 & 5 & 1\end{array}$

Figure 1. Kaplan-Meier estimate of overall survival (OS) and relapse-free survival (RFS).

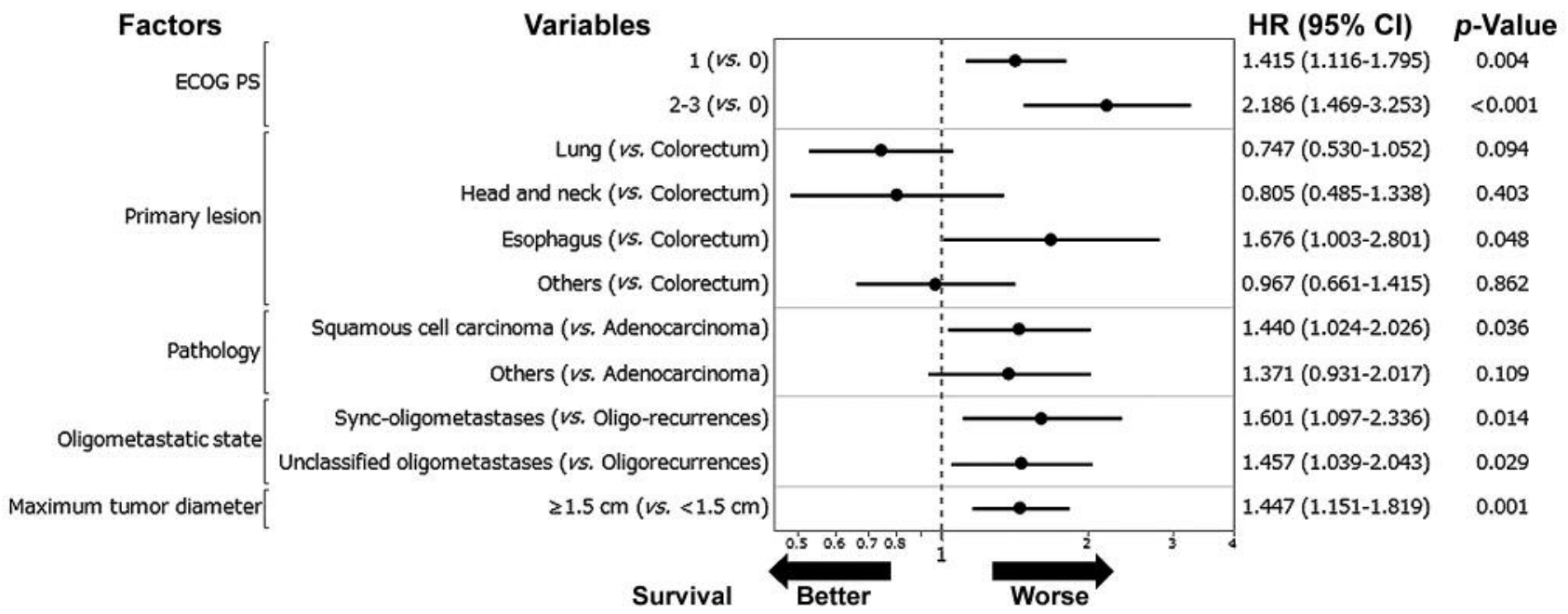

Figure 2. Multivariate analysis of overall survival. ECOG PS, Eastern Cooperative Oncology Group performance status; HR, hazard ratio; CI, confidence interval. 
Table II. Tumor and treatment characteristics.

\begin{tabular}{llc}
\hline Factors & & $\mathrm{n}(\%)$ \\
& & $\mathrm{N}=1,547$ \\
\hline SBRT date & $2004-2009$ & $468(34.0)$ \\
& $2010-2015$ & $910(66.0)$ \\
Maximum tumor diameter (cm) & Median, range & $1.5 ; 0.3-6.5$ \\
Number of metastases & 1 & $1012(73.9)$ \\
& $2-5$ & $358(26.1)$ \\
Field coplanarity & Coplanar field & $404(26.2)$ \\
& Non-coplanar field & $1139(73.8)$ \\
Dose prescription & IC & $1103(71.3)$ \\
& D95 of PTV & $317(20.5)$ \\
BED ${ }_{10}$ dose of IC (Gy) & Others & $127(8.2)$ \\
& Median, range & $105.6,75.0-289.6$ \\
OTT of SBRT (days) & $<100$ Gy & $173(13.7)$ \\
& $\geq 100$ Gy & $1093(86.3)$ \\
& Median, range & $7,3-81$ \\
\hline
\end{tabular}

SBRT, Stereotactic body radiotherapy; IC, isocenter; D95 of PTV, dose covering $95 \%$ of planning target volume; BED, biological effective; OTT, overall treatment time.

and $50.0 \%$, respectively) (7). Similarly, Yamashita et al. demonstrated that the median OS of lung oligo-recurrence was significantly higher compared to 66.6 that of syncoligometastases (66.6 vs. 23.9 months). Furthermore, in the same study, multivariate analysis indicated that lung oligorecurrences had significantly improved OS compared to that of sync-oligometastases (5). In a study that included 61 patients with brain NSCLC, pateients with oligometastases achieved a better OS comopared to patients with syncoligometastases. Moreover, a multivariate analysis indicated oligo-recurrence as a predictive factor for favorable prognosis $(\mathrm{HR}=0.253,95 \% \mathrm{CI}=0.082-0.043, p=0.025)$ (4). The current nationwide survey is consistent with these previous reports even with a large oligometastases population. A possible explanation for the superior OS of oligo-recurrence compared to that of sync-oligometastases, as reported in the aforementioned studies and the current one, is that patients with oligo-recurrence had controlled primary lesions and did not require further local therapy for their primary lesions. On the other hand, patients with sync-oligometastases must receive local therapy for both metastatic lesions and primary lesions. In general, primary lesions often invade the surrounding tissues and associated with metastases to the regional lymph nodes, thus, their LC is more difficult than that metastatic lesion (4-7).

Favorable prognostic factors to indicate surgery for pulmonary oligo-recurrence include a lung oligo-recurrence of osteosarcoma DFI $\geq 12$ months, 1 to 3 lung recurrent sites, and a total surgery lesion size of less than $30 \mathrm{~mm}$ (8). He et al. reported that in cancer patients with lung oligometastases from NSCLC, the median survival time of patients treated by
Table III. Univariate analyses for overall survival.

\begin{tabular}{|c|c|c|c|}
\hline Factors & $\mathrm{N}$ & MST & $p$-value \\
\hline \multicolumn{4}{|l|}{ Age } \\
\hline$<72$ & 683 & 49.3 & \\
\hline$\geq 72$ & 691 & 52.9 & 0.789 \\
\hline \multicolumn{4}{|l|}{ Gender } \\
\hline Male & 891 & 37.0 & \\
\hline Female & 483 & 53.7 & $<0.001$ \\
\hline \multicolumn{4}{|l|}{ ECOG PS } \\
\hline 0 & 742 & 60.3 & \\
\hline 1 & 473 & 42.0 & \\
\hline $2-3$ & 103 & 33.1 & $<0.001$ \\
\hline \multicolumn{4}{|l|}{ Primary lesion } \\
\hline Lung & 422 & 61.5 & \\
\hline Colorectum & 343 & 51.7 & \\
\hline Head and Neck & 113 & 52.8 & \\
\hline Esophagus & 113 & 27.1 & \\
\hline Others & 383 & 54.5 & $<0.001$ \\
\hline \multicolumn{4}{|l|}{ Pathology } \\
\hline Adenocarcinoma & 757 & 60.8 & \\
\hline Squamous cell carcinoma & 360 & 37.4 & \\
\hline Others & 186 & 56.0 & $<0.001$ \\
\hline \multicolumn{4}{|l|}{ Oligometastatic state } \\
\hline Oligo-recurrence & 1013 & 60.3 & \\
\hline Sync-oligometastases & 118 & 35.1 & \\
\hline Unclassified oligometastases & 120 & 37.6 & $<0.001$ \\
\hline \multicolumn{4}{|l|}{ SBRT date } \\
\hline 2004-2009 & 468 & 51.3 & \\
\hline $2010-2015$ & 906 & 53.6 & 0.762 \\
\hline \multicolumn{4}{|l|}{ Chemotherapy before SBRT } \\
\hline Yes & 501 & 47.9 & \\
\hline No & 863 & 51.6 & 0.175 \\
\hline \multicolumn{4}{|l|}{ Chemotherapy concurrent SBRT } \\
\hline Yes & 29 & 34.0 & \\
\hline No & 1345 & 51.7 & 0.102 \\
\hline \multicolumn{4}{|l|}{ Chemotherapy after SBRT } \\
\hline Yes & 190 & 37.9 & \\
\hline No & 878 & 56.0 & 0.033 \\
\hline \multicolumn{4}{|l|}{ Maximum tumor diameter, $\mathrm{cm}$} \\
\hline$<1.5$ & 595 & 61.5 & \\
\hline$\geq 1.5$ & 716 & 40.9 & $<0.001$ \\
\hline \multicolumn{4}{|l|}{ Number of metastases } \\
\hline 1 & 1009 & 51.7 & \\
\hline $2-5$ & 357 & 48.7 & 0.495 \\
\hline \multicolumn{4}{|l|}{ Field coplanarity } \\
\hline Coplanar field & 385 & 46.2 & \\
\hline Non-coplanar field & 985 & 52.6 & 0.421 \\
\hline \multicolumn{4}{|l|}{ Dose prescription } \\
\hline IC & 1011 & 48.8 & \\
\hline D95 of PTV & 252 & 60.9 & \\
\hline Others & 111 & NR & 0.433 \\
\hline \multicolumn{4}{|l|}{ BED10 dose of IC, Gy } \\
\hline$<100 \mathrm{~Gy}$ & 172 & 68.8 & \\
\hline$\geq 100 \mathrm{~Gy}$ & 1090 & 49.3 & 0.088 \\
\hline \multicolumn{4}{|l|}{ OTT of SBRT, day } \\
\hline$<7$ & 485 & 50.3 & \\
\hline$\geq 7$ & 580 & 56.0 & 0.994 \\
\hline
\end{tabular}

MST, Median survival time; ECOG, Eastern Cooperative Oncology Group; IC, isocenter; D95 of PTV, dose covering 95\% of planning target volume; NR, not reached; BED, biological effective dose; OTT, overall treatment time. 
surgery was higher than that of patients treated by systemic chemotherapy (18.2 vs. 9.1 months, $p<0.05$ ), although only 21 patients were included in that study (9). In another study of 7 patients who underwent hepatic resection for oligo-recurrence of NSCLC in the liver, the median survival time was 24.0 months (range $=15.2-30.2$ ), and 4 patients remained alive at the end of follow-up (23.4-30.2 months) (10). Lodeweges et al. reported that the 5-year OS of patients with pulmonary oligometastaes who underwent surgery for LC was similar to that of patients who underwent stereotactic ablative radiation therapy (SABR) (41\% and $45 \%$, respectively), suggesting SABR as a more effective and less invasive treatment method for pulmonary oligometastases; however, this was a small population-based retrospective study (11). Furthermore, a randomized phase II trial has suggested that OS of patients with oligometastastatic cancer treated by SABR was better than that of patients treated with palliative radiation therapy; however, only 99 patients were included in that study (12).

In the present nationwide survey of pulmonary oligometastases, oligo-recurrence, ECOG PS 0, adenocarcinoma pathology of the primary cancer, colorectal origin, and a smaller maximum tumor diameter $(<1.5 \mathrm{~cm})$ resulted in a significantly favorable prognosis for OS in the multivariate analysis. Although Niibe et al. (4) have previously shown that 5 -year OS rate was $0 \%$ in NSCLC patients with sync-oligometastases treated with stereotactic radiosurgery or stereotactic radiotherapy, the current study indicated that patients with sync-oligometastases reached the 5 -year OS. Despite the fact that the OS rate of the syncoligometastases group was inferior to that of the oligorecurrences group. The present result encourages patients, radiation oncologists, oncologic surgeons, medical oncologists and medical staff to treat sync-oligometastases aiming at cure or long-term survival.

The main limitation of the current study is its retrospective, non-randomized nature. The patients included were treated only in Japan. However, this is the first study to examine a large population database of 1,378 patients with pulmonary oligometastases.

In conclusion, this nationwide study as well as the studies mentioned above indicate that the OS of pulmonary oligo-recurrence is better than that the OS of syncoligometastases. The HR of sync-oligometastases $v s$. oligorecurrence was $1.601(95 \% \mathrm{CI}=1.097-2.336, p=0.014)$, and the adverse effects were manageable. Further studies, particularly large population-based prospective studies, will be required to construct more evident results supporting this hypothesis.

\section{Conflicts of Interest}

HO has grants from Accuray Japan, Cannon, present or during 36 months prior to publication.

\section{Author's Contributions}

NY drafted the protocol of this study, performed statistical analyses, wrote this manuscript and collected patients. TY revised protocol and performed statistical analyse, revised manuscript and collected patients. $\mathrm{KJ}, \mathrm{HO}, \mathrm{HY}, \mathrm{KK}$, AT revised protocol, revised manuscript and collected patients. YM, R-JO, MA, TS, KY, MK, MO, YM, KY, AN, HK, KY, TI, YT, KN, OS revised manuscript and collected patients.

\section{Acknowledgements}

The Authors would like to thank Drs. Akira Anbai, Gencho Kuga, Hajime Ikeda, Hideya Yamazaki, Hidekazu Tanaka, Hisashi Kaizu, Hitomi Kataoka, Hiroki Kobayashi, Ichiro Ogino, Imi Takanashi, Junichi Yokouchi, Kana Kobayashi, Katsuyuki Shirai, Keisuke Fujimoto, Koda Ryuichi, Kosuke Amano, Koutaro Terashima, Masaaki Yamashina, Masayoshi Yamada, Michiko Imai, Nanae Yamaguchi, Nobuhiko Yoshikawa, Nobuhisa Mabuchi, Saeko Hirota, Miyako Myojin, Satoshi Itasaka, Seiji Kubota, Shigetoshi Shimamoto, Shinichi Ogawa, Shinya Takemoto, Syuji Otsu, Takanori Fukuda , Takashi Sakamoto, Takashi Kawanaka, Takuya Yamazaki, Shizuko Ohashi, Susumu Kanazawa, Tetsuo Nonaka, Tetsuo Saito, Toshiki Kawamura, Toshinori Soejima, Tomoyasu Kumano, Toru Sakayauchi, Tsunehiko Kan, Yasuhiro Dekura, Yo Ushijima, Yoshiaki Okamoto, Yoshisuke Matsuoka, Yuko Shirata, Yumi Sato, Yu Takada, and Yutaka Naoi for their significant support. This study was also supported by a Grant-inAid from the Japanese Society for Radiation Oncology 2015-2016 and by JSPS KAKENHI Grant Number JP.17K10494.

\section{References}

1 Hellman S and Weichselbaum RR: Oligometastases. J Clin Oncol 13: 8-10, 1995. PMID: 7799047. DOI: 0.1200/JCO.1995.13.1.8

2 Niibe $\mathrm{Y}$ and Hayakawa K: Oligometastases and oligorecurrence: the new era of cancer therapy. Jpn J Clin Oncol 40: 107-111, 2010. PMID: 22047860. DOI:10.1093/jjco/hyp167

3 Niibe Y and Chang JY: Novel insights of oligometastases and oligo-recurrence and review of the literature. Pulm Med 2012: 261096, 2012. PMID: 229666429. DOI: 10.1155/2012/261096

4 Niibe Y, Nishimura T, Inoue T, Karasawa K, Shioyama Y, Jingu $\mathrm{K}$ and Shirato H: Oligo-recurrence predicts favorable prognosis of brain-only oligometastases in patients with non-small cell lung cancer treated with stereotactic radiosurgery or stereotactic radiotherapy: a multi-institutional study of 61 subjects. BMC Cancer 16: 659, 2016. PMID: 27542716. DOI: 10.1186/s12885016-2680-8

5 Yamashita H, Niibe Y, Yamamoto T, Katsui K, Jingu K, Kanazawa S, Terahara A and Nakagawa K: Lung stereotactic radiotherapy for oligometastases: comparison of oligo-recurrence and sync-oligometastases. Jpn J Clin Oncol 46: 687-691, 2016.PMID: 28219406. DOI: 10.1093/jjco/hyw047

6 Kanda Y: Investigation of the freely available easy-to-use software 'EZR' for medical statistics. Bone Marrow Transplant 48: 452458, 2013. PMID: 2320813. DOI: 10.1038/bmt.2012.244

7 Niibe Y, Yamashita H, Sekiguchi K, Takahashi W, Shiraishi K, Okuma K, Terahara A, Kawamori J and NakagawaK: Stereotactic body radiotherapy results for pulmonary oligometastases: a two-institution collaborative investigation. Anticancer Res 35: 4903-4908, 2015. PMID: 26254386 
8 Iwata $\mathrm{S}$, Yonemoto $\mathrm{T}$, Iizasa $\mathrm{T}$, Niibe $\mathrm{Y}$, Kamoda $\mathrm{H}$ and Ishii $\mathrm{T}$ : Oligo-recurrence of osteosarcoma patients: treatment strategies for pulmonary oligometastases. Ann Surg Oncol 22: S1332-1338, 2015.PMID: 26092699. DOI: 10.1245/s 10434-015-4682-1

9 He J, Li Y, An J, Hu L and Zhang J: Surgical treatment in nonsmall cell lung cancer with pulmonary oligometastases. World J Surg Oncol 15: 26, 2017. PMID: 2814827. DOI: 10.1186/s12957017-1105-8

10 Ishige F, Takayama W, Chiba S, Hoshino I, Arimitsu H, Yanagibashi $\mathrm{H}$ and Iwatate Y: Hepatectomy for oligo-recurrence of non-small cell lung cancer in the liver. Int J Clin Oncol 23: 647651, 2018. PMID: 295939. DOI: 10.1007/s10147-018-1262-y

11 Lodeweges JE, Klinkenberg TJ, Ubbels JF, Groen HJM, Langendijk JA and Wider J: Long-term outcome of surgery or stereotactic radiotherapy for lung oligometastases. J Thorac Oncol 12: 14421445, 2017. PMID: 28576747. DOI: 10.1016/j.jtho.2017.05.015
12 Palma DA, Olson R, Harrow S, Caede S, Louie AV, Haasbeek C, Mulroy L, Lock M, Rodrigues GB, Yaremko BP, Schellenberg D, Ahmad B, Griffioen G, Senthi S, Swaminath A, Kopek N, Liu M, Moore K, Currie S, Bauman GS, Wamer A and Senan S: Stereotactic ablative radiotherapy versus standard of care palliative treatment in patients with oligometastatic cancers (SABR-COMET): a randomized, phase 2, open-label trial. Lancet 393(10185): 2051-2058, 2019. PMID: 30982687. DOI: $10: 1016 / \mathrm{S} 140-6736(18) 32487-5$

Received October 30, 2019

Revised November 12, 2019

Accepted December 10, 2019 